\title{
Research Paper: Acute Kidney Injury in Poisoned Patients Admitted to ICU
}

\author{
Roghayeh Rashidi' ${ }^{1}$, Vahideh Mohamadzadeh² (D, Nasrin Milani ${ }^{\text {(D) }}$, Lida Jarahi ${ }^{4}$ (D), Bita Dadpour ${ }^{*}$ (iD) \\ 1. Pharmacological Research Center of Medicinal Plants, Mashhad University of Medical Sciences, Mashhad, Iran. \\ 2. Department of Pharmaceutical Nanotechnology, School of Pharmacy, Mashhad University of Medical Sciences, Mashhad, Iran. \\ 3. Department of Internal Medicine, Faculty of Medicine, Mashhad University of Medical Sciences, Mashhad, Iran. \\ 4. Department of Community Medicine, Faculty of Medicine, Mashhad University of medical sciences Mashhad Iran, Mashhad University of Medical \\ Sciences, Mashhad, Iran. \\ 5. Medical Toxicology Research Center, Faculty of Medicine, Mashhad University of Medical Sciences, Mashhad. Iran.
}

\begin{tabular}{|l|l|l}
$\begin{array}{l}\text { Use your device to scan } \\
\text { and read the article online }\end{array}$ & $\begin{array}{l}\text { ICU. International Journal of Medical Toxicology and Forensic Medicine. 2020; 10(4):30471. https://doi.org/10.32598/ijmtfm. } \\
\text { v10i4.30471 }\end{array}$ \\
dol'https://doi.org/10.32598/ijmttm.v10i4.30471
\end{tabular}

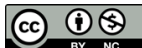

Article info:

Received: 15 May 2020

First Revision: 12 Jun 2020

Accepted: 18 Oct 2020

Published: 28 Nov 2020

\section{ABSTRACT}

Background: Acute Kidney Injury (AKI) is an abrupt decrease in kidney function, leading to the retention of urea and other nitrogenous waste products. Poisoned patients admitted to the Intensive Care Unit (ICU) may develop AKI due to some reasons. This study was done to evaluate the AKI in poisoned patients admitted to ICU.

Methods: 146 patients, admitted to the ICU of Imam Reza Hospital from March 2017 to March 2018 were studied. AKI status was assessed using Acute Kidney Injury Network (AKIN) and Risk, Injury, Failure, Loss of kidney function and End-stage kidney disease (RIFLE) classification. Data analysis was done through SPSS V. 22 software.

Results: Opioids, organophosphates, aluminum phosphide, multiple drugs, and other types of poisoning were the main five poisoning classes. Opioid toxicity was had the highest frequency with 51 patients; cases in this group experienced longer length of hospitalization stay and higher serum creatinine level than others did. Among 146 patients, 19 patients $(12.8 \%)$ died, and 97 patients $(66 \%)$ were transferred to the ICU. Of all cases, 18 patients $(12.3 \%)$ had renal dysfunction (six patients were at risk, five patient at injury, and seven patients were at failure phase based on the RIFLE criteria). Renal replacement therapy was required in 24 cases (16.4\%).

Conclusion: It is unlikely to detect a significant difference in the occurrence of AKI between the main poisoning classes. Being the largest group of intoxicated patients admitted to the ICU, the opioid poisoning had the highest rate of AKI
Acute kidney injury,

Poisoning, ICU

\section{* Corresponding Author:}

Bita Dadpour, PhD.

Address: Department of Clinical Toxicology, Imam Reza Hospital, Medical Toxicology Center, Mashhad University of Medical Sciences, Mashhad, Iran Tel: +98 (915) 5149842

E-mail:dadpourb@mums.ac.ir 


\section{Introduction}

A

cute Kidney Injury (AKI) is an abrupt reduction in kidney function, leading to impairment of renal capacity to stabilize metabolic balance. There are three main categories of AKI: Prerenal, intrinsic, and post renal AKI. Volume depletion, preglomerular vasoconstriction, systemic vasodilation, or heart failure can induce renal hypoperfusion, and consequently, sequences of events may be initiated due to decreased urinary sodium excretion. This phenomenon may occur when a toxic exposure compromises renal perfusion, like anticoagulant toxicity, followed by bleeding or diuretic toxicity leading to volume depletion. Renal parenchyma may be harmed by glomerular, vascular, or tubulointerstitial etiologies, which is considered as renal AKI [1-3].

Clopidogrel and quinine are two examples of xenobiotics resulting in thrombotic microangiopathy. The nephrotic or nephritic syndrome may be the presentation of xenobiotic-induced glomerular disorders. Xenobiotic glomerular lesions may release antigens into circulation leading to antigen-antibody complex formation. The deposition of these complexes in the basement membrane of glomeruli changes its properties. They can also affect the balance of the immunoregulatory system. Cefixime, insect venom, heroin, and cocaine can be referred to as examples of agents that can cause nephrotic syndrome [2].

Acute Tubular Necrosis (ATN) is the most common etiology of AKI in hospitalized cases. ATN arises after a toxic or ischemic injury. It presents as a rapid progression of kidney failure. Oliguria is followed by most forms of ATN; nonetheless, AKI due to aminoglycosides exposure is typically nonoliguric. Antibacterials, such as aminoglycosides and colistin, aluminum phosphide, and paraquat are some xenobiotics that cause ATN. AKI is common after severe paraquat poisoning and usually exhibits a fatal outcome $[4,5]$.

Increased urinary concentration of myoglobin (due to rhabdomyolysis) or kidney hypoperfusion leads to the inspissation of myoglobin in the tubular lumen causing toxicity. Trauma-induced muscle injury, prolonged immobilization, and opioids and sedative-hypnotic drugs, which cause prolonged and deep loss of consciousness, severe muscle contraction due to stimulant drugs, such as amphetamine compounds, and tonic-clonic seizures, snake venom [6], and hyperthermia are the most common etiologies in case of rhabdomyolysis [7].
The third category of AKI is post-renal. Several xenobiotics can cause obstruction. Anticholinergic agents, which impair bladder contraction, are the most responsible drugs. Intratubular obstruction due to oxalosis and crystalluria is the proposed mechanism for post-renal AKI following ethylene glycol poisoning. When a physician faces a patient presenting with an acutely deteriorating renal function, it often poses a difficult challenge for etiology diagnosis owing to the possibility of several factors involved [2].

There are no criteria to identify the numerous etiologies that cause AKI. It is incorrect to treat AKI as a single disease, particularly grouping with hemodynamic causes (i.e. "prerenal AKI") and acute tubular necrosis.

Although different causes of AKI are associated with different long-term outcomes and prognoses, the severity of AKI stage is correlated with mortality risk and Intensive Care Unit (ICU) and hospital length of stay [8].

\section{Materials \& Methods}

The present retrospective study was done to evaluate AKI prevalence in vulnerable poisoned patients admitted to the ICU of teaching Imam Reza Hospital from March 2017 to March 2018. Data were collected from the records of 146 patients recorded in the ICU of this academic hospital as the only referral center for poisoning in Mashhad, northeastern Iran as a metropolitan city. The Incidence of poisoning with different drugs, pesticides, and illegal substances was also regarded in the study period. Serum Creatinine (Cr) was evaluated during admission and then every $48 \mathrm{~h}$. Gradual serum $\mathrm{Cr}$ loss was discovered during this period.

\section{Demographics of the patients}

This routine data-based study achieved the approval of the Ethics Committee (IR.MUMS.fm.REC.1396.499) of Mashhad University of Medical Sciences to evaluate Serum $\mathrm{Cr}$ and urine output of 146 healthy patients admitted due to poisoning by at least one of the poisonous substances, including opioids, organophosphorus, aluminum phosphide, psychiatric drugs (benzodiazepine, antipsychotics, phenobarbital, acetaminophen, tricyclic antidepressants (TCAs), and unknown drugs), juice suspected to be contaminated with drugs, Carbon Monoxide (CO), paraquat, botulinum toxin, methanol, methamphetamine, and ethanol. 
Table 1. Risk, Injury, Failure, Loss of kidney function and End-stage kidney disease (RIFLE) classification and the Acute Kidney Injury Network (AKIN) classification/staging system of acute kidney injury

\begin{tabular}{|c|c|c|c|c|}
\hline \multirow[b]{2}{*}{ Class } & \multicolumn{2}{|l|}{ RIFLE } & \multicolumn{2}{|l|}{ AKIN } \\
\hline & GFR & Uo & GFR & UO \\
\hline $\begin{array}{l}\text { Risk (RIFLE) or stage } 1 \\
\text { (AKIN/KDIGO) }\end{array}$ & $\uparrow \mathrm{SCr} \times 1.5$ or $\downarrow$ GFR $>25 \%$ & $<0.5 \mathrm{~mL} / \mathrm{kg} / \mathrm{h} \times 6 \mathrm{~h}$ & $\begin{array}{l}\uparrow S C r \geq 26.5 \mu \mathrm{mol} / \mathrm{L} \\
(\geq 0.3 \mathrm{mg} / \mathrm{dL}) \text { or } \uparrow \mathrm{SCr} \\
\geq 150 \text { a } 200 \%(1.5 \text { a } 2 \times)\end{array}$ & $<0.5 \mathrm{~mL} / \mathrm{kg} / \mathrm{h} \times 6 \mathrm{~h}$ \\
\hline $\begin{array}{l}\text { Injury (RIFLE) or stage } \\
2 \text { (AKIN/KDIGO) }\end{array}$ & $\uparrow \mathrm{SCr} \times 2$ or $\downarrow$ GFR $>50 \%$ & $<0.5 \mathrm{~mL} / \mathrm{kg} / \mathrm{h} \times 12 \mathrm{~h}$ & $\uparrow \mathrm{SCr}>200$ a $300 \%$ (>2 a $3 \times)$ & $<0.5 \mathrm{~mL} / \mathrm{kg} / \mathrm{h} \times 12 \mathrm{~h}$ \\
\hline $\begin{array}{l}\text { Failure (RIFLE) or stage } \\
3 \text { (AKIN/KDIGO) }\end{array}$ & $\begin{array}{l}\uparrow \mathrm{SCr} \times 3 \text { or } \downarrow \mathrm{GFR}>75 \% \\
\text { or if baseline } \mathrm{SCr} \geq 353.6 \\
\mu \mathrm{mol} / \mathrm{L}(\geq 4 \mathrm{mg} / \mathrm{dL}) \uparrow \mathrm{SCr} \\
>44.2 \mu \mathrm{mol} / \mathrm{L}(>0.5 \mathrm{mg} / \mathrm{dL})\end{array}$ & $\begin{array}{l}<0.3 \mathrm{~mL} / \mathrm{kg} / \mathrm{h} \times 24 \mathrm{~h} \\
\quad \text { or anuria } \times 12 \mathrm{~h}\end{array}$ & $\begin{array}{l}\uparrow \mathrm{SCr}>300 \% \text { (>3x) or if base- } \\
\text { line } \mathrm{SCr} \\
\geq 353.6 \mu \mathrm{mol} / \mathrm{L}(\geq 4 \mathrm{mg} / \mathrm{dL}) \\
\uparrow \mathrm{SCr} \\
\geq 44.2 \mu \mathrm{mol} / \mathrm{L}(\geq 0.5 \mathrm{mg} / \mathrm{dL})\end{array}$ & $\begin{array}{c}<0.3 \mathrm{~mL} / \mathrm{kg} / \mathrm{h}(24 \mathrm{~h}) \\
\text { oranuria }(12 \mathrm{~h})\end{array}$ \\
\hline $\begin{array}{l}\text { Loss of kidney func- } \\
\text { tion (RIFLE) }\end{array}$ & $\begin{array}{l}\text { Complete loss of kidney } \\
\text { function }>4 \text { weeks }\end{array}$ & & & \\
\hline $\begin{array}{l}\text { End-stage kidney } \\
\text { disease (RIFLE) }\end{array}$ & $\begin{array}{l}\text { Complete loss of kidney } \\
\text { function }>3 \text { months }\end{array}$ & & & \\
\hline
\end{tabular}

GFR, glomerular filtration rate; UO, urine output; SCr, serum creatinine; AKIN, Acute Kidney Injury Network; KDIGO, Kidney Disease: Improving Global Outcomes

\section{Inclusion and exclusion criteria}

Inclusion criteria were Iranian nationality living in different regions and provinces, the age of over 18 years, and no history of renal dysfunction, end-stage renal disease, and kidney transplantation. Patients with previous comorbidities, like diabetes mellitus, hypertension, or known kidney diseases were excluded.

\section{Methods of measurement}

A checklist was initially developed to collect the demographic data, including age, primary diagnosis, comorbid disease, laboratory tests on admission, urea and serum $\mathrm{Cr}$ (once on admission and every $48 \mathrm{~h}$ ), need for hemodialysis, length of ICU stay, and outcomes of all patients. Of 411 patients admitted to ICU within a year, 146 cases who met the inclusion criteria were selected. The basic laboratory investigation, including serum $\mathrm{Cr}$ and urea, complete blood count, liver function test, serum lactate dehydrogenase, and urinalysis were carried out for all patients from the first day of admission and then every other day. Moreover, previous serum $\mathrm{Cr}$, when available, was recorded as baseline $\mathrm{Cr}$.

Risk, Injury, Failure, Loss of kidney function and End-stage kidney disease (RIFLE) classification was suggested to define and categorize the severity of AKI. It is associated with sudden changes in serum $\mathrm{Cr}$ (1-7 days), Glomerular Filtration Rate (GFR), and/ or sustained depletion of urine output (persisting $>24$ h) (Table 1). The RIFLE classification is available in clinical settings to classify AKI in different settings of hospitalized patients and is an acceptable predictor of mortality and morbidity [9]. When the baseline serum $\mathrm{Cr}$ is known, this criterion can be utilized conveniently. However, it is unknown to a considerable number of patients. In such cases, it should be calculated using the Modification of Diet in Renal Disease (MDRD) [10], providing a baseline GFR of $75 \mathrm{~mL} / \mathrm{min} / 1.73 \mathrm{~m}^{2}$.

A modified version of the RIFLE was proposed to enhance the efficiency of AKI diagnosis with regard to Acute Kidney Injury Network (AKIN) classification [11]. Although AKIN criteria could improve the sensitivity of the AKI diagnosis, it does not seem to be significant for RIFLE classification in predicting hospital mortality of critically ill patients [12].

The definition of a baseline $\mathrm{Cr}$ for people without a baseline measurement of serum $\mathrm{Cr}$ is one of the constraints in measuring the variations in serum $\mathrm{Cr}$ [13] Thus, the authors of the RIFLE criterion had proposed back-calculation of an expected baseline serum $\mathrm{Cr}$ accumulation through the four-variable MDRD equation, considering a $75 \mathrm{~mL} / \mathrm{min} / 1.73 \mathrm{~m}^{2}$ baseline for GFR [2]. The baseline serum $\mathrm{Cr}$ of more than $1 \mathrm{mg} /$ $\mathrm{dL}$ was considered for females and above $1.3 \mathrm{mg} / \mathrm{dL}$ for males. AKI was considered as a 1.5 -time increase of baseline in serum $\mathrm{Cr}$, an increase in serum $\mathrm{Cr}$ by $\geq 0.3$ $\mathrm{mg} / \mathrm{dL}$ from baseline $(\geq 26.5 \mu \mathrm{mol} / \mathrm{L})$, or a reduction in urine output by $<0.5 \mathrm{~mL} / \mathrm{kg} / \mathrm{h}$ for 6 to $12 \mathrm{~h}$. Regardless of whether the subjected were poisoned, when growth in baseline serum $\mathrm{Cr}$ was evident, the previous serum $\mathrm{Cr}$ 
Table 2. Prevalence of Acute Kidney Injury (AKI) in different types of poisoning

\begin{tabular}{|c|c|c|c|c|c|c|}
\hline Toxic Agent & Total number & Dialysis & Risk & Injury & Failure & Deceased Cases \\
\hline Opioids & 51 & 11 & 1 & 2 & 2 & 4 \\
\hline Aluminum phosphide & 12 & 1 & & - & 1 & 2 \\
\hline Organophosphate & 18 & 1 & - & - & - & - \\
\hline Multiple drug & 27 & - & 5 & 1 & 1 & 5 \\
\hline Phenobarbital & 1 & 1 & - & - & - & - \\
\hline Benzodiazepine & 8 & 1 & - & 1 & - & 3 \\
\hline Acetaminophen & 1 & 1 & - & & 1 & 1 \\
\hline Tricyclic Antidepressants (TCA) & 1 & - & - & - & - & - \\
\hline Methanol & 2 & 2 & - & - & - & - \\
\hline Lead & 9 & 1 & - & - & - & 1 \\
\hline Botulinum toxin & 3 & - & - & - & 1 & - \\
\hline Unknown poison & 4 & 1 & - & - & 1 & - \\
\hline $\mathrm{CO}$ & 4 & 2 & - & 1 & - & 1 \\
\hline Methamphetamine & 2 & - & - & - & - & - \\
\hline Ethanol & 1 & 1 & - & - & - & - \\
\hline Paraquat & 1 & 1 & - & - & - & 1 \\
\hline Depilating powder & 1 & - & - & - & - & 1 \\
\hline Total & 146 & 24 & 6 & 5 & 7 & 19 \\
\hline
\end{tabular}

was considered as a baseline to evaluate the incidence of renal failure.

\section{Method of implementation}

The Clinical Toxicology Department at Imam Reza Hospital (p) is the only center in eastern Iran to admit all the poisoned patients from the surrounding towns and villages.

Patients' demographic information, hospitalization date, initial diagnosis, concurrent diseases, serum $\mathrm{Cr}$, duration of need for mechanical ventilation, and the need for vasopressor medications were recorded daily until discharge from ICU. The length of stay in the ICU and hospital and hospital mortality were also noted. Nephrology consultation was considered for every patient suspected of AKI. The patients' kidney function was assessed and the dosage of antidote was administered proportionately. A proper dose of medication was dispensed in renal impairment to prevent adverse reactions in the kidney. Besides, other nephrotoxic medicines, such as antibiotics were avoided.

Kruskal-Wallis test was used to compare the length of hospitalization (which is a quantitative variable with statistically abnormal distribution) among the groups. Instead of the mean and standard deviation, the 50th, 25th, and 75 th percentiles were used to determine the duration of hospitalization, the shortest and the longest stays in each group. Because the distribution of hospitalization length was abnormal, the median was used along with the 25 th and 75 th percentiles (interquartile range). Data were analyzed using SPSS software version 22. Moreover, Mann-Whitney U, Kruskal-Wallis, and chi-square tests were applied for data analysis. The significance level was also considered to be less than 0.05 . 
Table 3. Kidney condition of poisoned patients on admission in an Intensive Care Unit (ICU)

\begin{tabular}{|c|c|c|}
\hline Kidney Condition & Frequency & Percent \\
\hline Kidney at risk phase & 6 & 4.1 \\
\hline Kidney at injury phase & 5 & 3.4 \\
\hline Kidney at failure phase & 7 & 4.7 \\
\hline Total & 18 & 12.2 \\
\hline
\end{tabular}

\section{Results}

A total of 146 poisoned cases, 45 women and 101 men were recruited in the present study. The mean age of patients was 44 years.

Patients were admitted due to opioid poisoning (51), aluminum phosphide poisoning (12), organophosphate poisoning (18), drug poisoning (benzodiazepine (8), phenobarbital (1), TCA (1), acetaminophen (1), multiple drugs (27)) other types of poisoning (methanol toxicity (2), lead poisoning (9), botulinum toxin (3), unknown poisoning (4), Co poisoning (4), methamphetamine toxicity (2), ethanol toxicity (1), paraquat toxicity (1), and depilating powder toxicity (Zarnikh) (1).

Table 2 presents kidney function in different poisoned patients admitted to ICU. Subsequently, AKI status on admission was assessed using AKIN and RIFLE classifications.

As can be seen in the table, about one-third of the cases were in the opioid poisoning class and about $20 \%$ needed hemodialysis. On admission, one case was in the risk phase of kidney damage and four cases were in the injury and failure phase (two cases in each phase).

Hemodialysis was performed in solely one case among all the 12 cases with aluminum phosphide poisoning.
A similar pattern was observed in organophosphate poisoned cases, among which merely one case needed hemodialysis. Also, of seven cases admitted due to multiple drug poisoning in different cases with ACI (5 cases in risk and 2 cases in injury and failure phase (one case in each phase)), none needed hemodialysis. However, the only case with phenobarbital poisoning as well as the only case with paraquat toxicity received hemodialysis. Both cases with methanol toxicity and one out of the nine cases with lead poisoning needed hemodialysis, while none of them were in the injury phase on admission. Two cases of all four CO poisoned patients underwent hemodialysis, whereas one case was in the injury phase on admission.

Among all 146 patients, 13\% (19 patients) died during the first $48 \mathrm{~h}$ after admission, 4.1\% (6 patients) were discharged with informed consent, and 66\% (97 patients) were transferred to the ICU.

Renal replacement therapy was required in 24 cases (16.4). Of all cases, 18 patients $(12.3 \%)$ had renal dysfunction on admission, six patients were at risk, five patients were at the injury phase, and seven patients were at the failure phase based on the RIFLE criteria (Table 3 ).

According to the results, the maximum serum $\mathrm{Cr}$ in the opioid poisoning group was $11.6 \mathrm{mg} / \mathrm{dL}$ and

Table 4. occurrence of dialysis among patients with RIFLE criteria.

\begin{tabular}{cccc}
\hline \multirow{2}{*}{ RIFLE criteria } & Dialysis & Yes & Total \\
\cline { 2 - 3 } & No & $2(33.3 \%)$ & $6(100 \%)$ \\
\hline Risk & $4(66.7 \%)$ & $2(40 \%)$ & $5(100 \%)$ \\
\hline Failure & $3(60 \%)$ & $4(57.1 \%)$ & $7(100 \%)$ \\
\hline Total & $3(42.9 \%)$ & $8(44.4 \%)$ & 18 (100\%) \\
\hline & $10(55.6 \%)$ & & International Journal of \\
Medical Toxicology E Forensic Medicine
\end{tabular}


occurred on the fifth day. The maximum serum $\mathrm{Cr}$ in the organophosphorus poisoning group was $4.5 \mathrm{mg} / \mathrm{dL}$ and took place on the eighth day and the maximum serum $\mathrm{Cr}$ in the phosphide poisoning group was $3.4 \mathrm{mg} / \mathrm{dL}$ and it was evident on the first day. Also, the maximum serum $\mathrm{Cr}$ in the drugs group occurred on day five and was $4.7 \mathrm{mg} / \mathrm{dL}$, while the maximum $\mathrm{Cr}$ level in patients poisoned with different types of poisoning was $6.8 \mathrm{mg} /$ $\mathrm{dL}$ detected on day five.

Table 4 presents the data on the prevalence of dialysis among patients with RIFLE criteria. Interestingly, the average need for dialysis is approximately the same in cases in the risk and injury phases, (about 33.3\% and $40 \%$, respectively), whereas this percentage was higher among cases in the failure phase, which was around $57 \%$.

In terms of the need for mechanical ventilation in different groups, this rate in the opioid group was $21.6 \%$, in the organophosphorus group was $47.1 \%$, in the phosphide group was $27.3 \%$, in the drug group was $57.1 \%$, and in patients with different types of poisoning was $21.4 \%$.

It was noticeable that $90.2 \%$ of the cases in the opioid group, $94.1 \%$ of organophosphorus poisoning cases, $63.6 \%$ of phosphide poisoning group, $100 \%$ of drug toxicity group, and $93.5 \%$ of patients with different types of poisoning did not need vasopressor.

There was a significant difference between the groups regarding the number of hospitalization days (PV: 0.019). The lowest number was found in the aluminum phosphide poisoning group, and the highest in opioid poisoned cases.

Patients poisoned with phenobarbital and methanol received hemodialysis. On the contrary, patients poisoned with methadone and botulinum toxin did not have to undergo dialysis.

Mann-Whitney U test was used to discover the relation between the need for dialysis and RIFLE criteria and based on the results, there was no correlation between them (PV: 0.24). Also, there was no correlation between the occurrence of AKI and mortality rate (PV: 0.31), length of hospitalization, and the need for vasopressor administration, and mechanical ventilation (PV: 0.08, 0.23 , and 0.17 ).

\section{Discussion}

In spite of considerable efforts to raise awareness in the general public, some influential components, like accessibility and inexpensiveness of poisons along with social problems and the growing trend of emotional control and impulsiveness in the industrialized world prevent the rate of some acute poisonings from a noticeable decrease or even increase. Acute poisoning may cause damages to different organs, such as the kidney.

Early and thorough treatment of acute poisoning can ensure full recuperation in many cases. Assessing the severity of the disease together with distinguishing the patients who require more consideration is an efficient measure, which expands the likelihood of patient survival. There exists a tremendous amount of research about AKI in patients with the acute condition; however, presently there is only a small number of investigations assessing kidney injury and its pertaining outcome in poisoned patients $[2,9]$. AKI may occur as part of multiple organ damage following acute toxicity, which can in turn lead to a notable risk of short- and long-run death [4, 14, 15]. The crude rate of death and length of ICU stay is increased with the seriousness of AKI $[9,16]$. The results of our study were indicative of a noticeable contrast between the groups when it comes to the length of hospitalization ( $\mathrm{PV}=0.019)$ in light of the Kruskal-Wallis test; as a consequence, AKI would be costlier.

Patients poisoned with aluminum phosphide had the shortest hospitalization period, while those poisoned with opioids had the longest. This time bracket had a standard deviation of between 4 and 21 days with a mean of 8 days. Acute aluminum phosphide poisoning is a rapidly progressive illness and opioid poisoning cases are often chronic abusers and suffer from different organ dysfunctions most of the time.

Opioid poisoning cases accounted for the largest number of included subjects. Of 51 cases admitted due to this poisoning, nine cases had AKI on admission. Of the 42 remaining cases, four cases experienced AKI in the first $48 \mathrm{~h}$ of admission. About one-fourth of the patients needed hemodialysis, while the lower remaining experienced risk, injury, and failure phases of kidney damage with a roughly equivalent rate. Previous investigations have demonstrated a correlation between opioid consumption and kidney disorders. The kidney damage caused by such substances derives from a myriad of mechanisms leading to an alteration in renal excretion of water and sodium $[17,18]$. Rhabdomyolysis, volume 
changes, amyloidosis, and renal lipidosis triggered by opioids toxicity may lead to a wide range of kidney disorders $[19,20]$. Prerenal and postrenal AKI and acute tubular necrosis may all occur in the context of opioid toxicity [17].

Speaking of acute aluminum phosphide poisoning, which results in quick development to several organ failures, the patient may die prior to the occurrence of intense kidney failure. In the current investigation, of all 12 patients with aluminum phosphide poisoning, seven cases had initially normal kidney function and one case developed renal failure on the second day. Additionally, two cases died and one case underwent hemodialysis.

Evidence suggests that organophosphate poisoning correlates with enhanced risk of AKI [14, 21]. However, the recurrence of dialysis in such patients was not so high, and none of them met the RIFLE criteria.

In this survey, of 18 cases with organophosphate poisoning one needed hemodialysis. Of the 27 patients with multi-drug poisoning, five cases (19.2\%) died and 18 patients $(69.2 \%)$ were transferred to the ward. Four patients $(15.4 \%)$ underwent hemodialysis. Of the seven cases who had $\mathrm{Cr}$ rise on admission, five cases were at risk phase, one patient was at injury and one patient was at the failure phase.

Of all 22 survived patients, 15 had normal kidney function on admission, and one suffered from AKI manifestation by day two.

As a complication of some drugs, such as nonsteroidal anti-inflammatory drugs (NSAIDs), AKI may occur at the end phase of toxicity conditions in most drug poisonings and as a part of multi-organ failure [22, 23].

There was a vast variety of poisonings in the miscellaneous group. For example, there were two cases with methanol toxicity who both underwent hemodialysis. Metabolic acidosis and renal failure are frequently followed by acute massive methanol toxicity [24, 25]. Moreover, there were two patients with methamphetamine (Shisheh in Iran) toxicity, both of whom were discharged and did not need hemodialysis. ATN is a known complication of amphetamine and methamphetamine toxicity and varying mechanisms have been proposed for it $[26,27]$. Among three cases with the diagnosis of botulism, one died and none received hemodialysis. Respiratory failure is the main cause of death in cases with botulism and AKI is not expected as a usual manifestation [28].
There were nine cases of lead poisoning, of whom one died and one underwent hemodialysis. Lead nephropathy is the known organ damage following lead toxicity for several reasons and mechanisms [29, 30]. There were four cases of unknown poisoning with no death and one needed hemodialysis.

One case of ethanol toxicity was admitted who did not undergo hemodialysis and deceased. Of four patients diagnosed with $\mathrm{CO}$ poisoning, one died and two needed hemodialysis. AKI may occur following $\mathrm{CO}$ poisoning. Youn-Jung Kim et al. reported that AKI following CO poisoning is mostly evident within $24 \mathrm{~h}$ after $\mathrm{CO}$ poisoning. The occurrence of AKI is correlated with poor outcomes [20]. There was a case of paraquat toxicity who underwent hemodialysis and deceased. Kidneys and lungs are two main organs affected by acute fatal poisoning by herbicide paraquat $[4,5]$; there was a case of severe acetaminophen poisoning who received hemodialysis and deceased. AKI may have been occurred in about $50 \%$ of the cases with overt hepatic failure following acetaminophen toxicity $[31,32]$; there was one case with arsenic toxicity following oral consumption of depilating powder (Zarnich powder) who did not need hemodialysis and died. Refractory hypotension, nausea and vomiting, the cardiac manifestation that mimic myocardial infarction, and Systemic Inflammatory Response Syndrome (SIRS), and ATN are expected in acute arsenic toxicity [33].

Patients were studied as soon as they admitted to the ICU. There was insufficient information about the medical history of some patients. Consequently, if patients had high serum $\mathrm{Cr}$ on admission, the severity of symptoms could not be determined in order to exclude them from the study. Nonetheless, having experienced a significant improvement in general conditions in the course of hospitalization, their previous status was considered as an acute condition.

The other limitation can be associated with the regional focus and investigating the population accessible in Mashhad only. Moreover, the type and amount of toxic substances were determined based on history taken from the patients or relatives and/or urine qualitative screen tests. As a result, some percentages mentioned by patients could not describe the precise amount of substance taken. Alternatively, the screening test was inaccessible for many drugs and substances in our setting; therefore, it was difficult to ascertain the dosage of drug or medicine that could cause renal damage. Also, the majority of patients exposed to poisons were discharged from the hospital. Due to defective referral 
and family medicine follow-up in the health care system nation-wide, the detection of renal involvement, in the long run, was impossible.

\section{Conclusion}

This study revealed the possibility of renal involvement and AKI with the ingestion of different poisonous substances, which usually have other uses. Most of such materials described here were both easily available and inexpensive. The evaluation of renal damage by more common forms of poisoning is yet open to debate and requires further research. Owing to the expansive range of symptoms in all types of poison groups, achieving a meaningful outcome seems to be unlikely. Due to completely unclear information reported by patients about the time of the poisoning, a more comprehensive study is recommended. The findings are assumed to be beneficial to intensivists, clinical toxicologists, and particularly nephrologists, in managing such cases.

\section{Ethical Considerations}

\section{Compliance with ethical guidelines}

All ethical principles were considered in this article. The participants were informed about the purpose of the research and its implementation stages; they were also assured about the confidentiality of their information; Moreover, They were allowed to leave the study whenever they wish, and if desired, the results of the research would be available to them.

\section{Funding}

This research did not receive any specific grant from funding agencies in the public, commercial, or non-profit sectors.

\section{Author's contributions}

All authors contributed to designing, running, and writing all parts of the research.

\section{Conflict of interest}

The authors declared no conflict of interest.

\section{Acknowledgements}

The authors appreciate the insightful cooperation of the ICU department staff and also Prof. Babak Mostafazadeh for their cooperation in the research.

\section{References}

[1] Bellomo R, Kellum JA, Ronco C. Acute kidney injury. Lancet. 2012; 380(9843):756-66. [DOI:10.1016/S0140-6736(11)61454-2]

[2] Brent J, Burkhart K, Dargan P, Hatten B, Megarbane B, Palmer $\mathrm{R}$, et al. Critical care toxicology: Diagnosis and management of the critically poisoned patient. Amsterdam: Springer; 2017. [DOI:10.1007/978-3-319-17900-1_76] [PMID]

[3] Levey AS, James MT. Acute kidney injury. Ann Intern Med. 2017; 167(9):Itc66-itc80. [DOI:10.7326/AITC201711070] [PMID]

[4] Mohamed F, Endre Z, Jayamanne S, Pianta T, Peake P, Palangasinghe $C$, et al. Mechanisms underlying early rapid increases in creatinine in paraquat poisoning. PLoS One. 2015; 10(3):e0122357. [DOI:10.1371/journal.pone.0122357] [PMID] [PMCID]

[5] Weng CH, Chen HH, Hu CC, Huang WH, Hsu CW, Fu JF, et al. Predictors of acute kidney injury after paraquat intoxication. Oncotarget. 2017; 8(31):51345-54. [DOI:10.18632/oncotarget.17975] [PMID] [PMCID]

[6] Gopalakrishnan N. Snake envenoming horizontal line an underreported cause of acute kidney injury. Kidney Int Rep. 2019; 4(5):643-6. [DOI:10.1016/j.ekir.2019.03.014] [PMID] [PMCID]

[7] Glisson SN. Malignant hyperthermia. Compr Ther. 1988; 14(10):33-41.

[8] Ali T, Khan I, Simpson W, Prescott G, Townend J, Smith W, et al. Incidence and outcomes in acute kidney injury: A comprehensive population-based study. J Am Soc Nephrol. 2007; 18(4):1292-8. [DOI:10.1681/ASN.2006070756] [PMID]

[9] Ricci Z, Cruz D, Ronco C. The RIFLE criteria and mortality in acute kidney injury: A systematic review. Kidney Int. 2008; 73(5):538-46. [DOI:10.1038/sj.ki.5002743] [PMID]

[10] Manjunath G, Sarnak MJ, Levey AS. Prediction equations to estimate glomerular filtration rate: An update. Curr Opin Nephrol Hypertens. 2001; 10(6):785-92. [DOI:10.1097/00041552-200111000-00009] [PMID]

[11] Lopes JA, Jorge S. The RIFLE and AKIN classifications for acute kidney injury: A critical and comprehensive review. Clin Kidney J. 2013; 6(1):8-14. [DOI:10.1093/ckj/sfs160] [PMID] [PMCID]

[12] Jiang F, Chen YH, Liang XL, Xu LX, Ma GP, Hu PH, Liu SX, Shi W. The sensitivity and accuracy of RIFLE and AKIN criteria for acute kidney injury diagnosis in intensive care unit patients. Zhongguo wei zhong bing ji jiu yi xue $=$ Chinese critical care medicine $=$ Zhongguo weizhongbing jijiuyixue. 2011; 23(12):759-62. https://europepmc.org/article/ med/22153016

[13] Bellomo R, Ronco C, Kellum JA, Mehta RL, Palevsky P. Acute renal failure-definition, outcome measures, animal models, fluid therapy and information technology needs: the Second International Consensus Conference of the Acute Dialysis Quality Initiative (ADQI) Group. Crit Care. 2004; 8(4):R204.

[14] Yadla M, Sailaja S, Ahmed N, Uppin M, Arlappa N. An unusual case of insecticide poisoning presenting as acute kidney injury. Saudi J Kidney Dis Transpl. 2017; 28(6):1432-4. [DOI:10.4103/1319-2442.220867] [PMID] 
[15] Forni LG, Darmon M, Ostermann M, Oudemans-van Straaten HM, Pettila V, Prowle JR, et al. Renal recovery after acute kidney injury. Intensive Care Med. 2017; 43(6):855-66. [DOI:10.1007/ s00134-017-4809-x] [PMID] [PMCID]

[16] Garzotto F, Piccinni P, Cruz D, Gramaticopolo S, Dal Santo M, Aneloni G, et al. RIFLE-based data collection/ management system applied to a prospective cohort multicenter Italian study on the epidemiology of acute kidney injury in the intensive care unit. Blood Purif. 2011;31(1-3):15971.[DOI:10.1159/000322161] [PMID]

[17] Mallappallil M, Sabu J, Friedman EA, Salifu M. What do we know about opioids and the kidney? Int J MolSci. 2017; 18(1):223. [DOI:10.3390/ijms18010223] [PMID] [PMCID]

[18] Mercadante S, Arcuri E. Opioids and renal function. J Pain. 2004; 5(1):2-19. [DOI:10.1016/j.jpain.2003.09.007] [PMID]

[19] Alinejad S, Ghaemi K, Abdollahi M, Mehrpour O. Nephrotoxicity of methadone: A systematic review. Springerplus. 2016; 5(1):2087. [DOI:10.1186/s40064-016-37571] [PMID] [PMCID]

[20] Kim YJ, Sohn CH, Seo DW, Oh BJ, Lim KS, Chang JW, Kim WY. Analysis of the development and progression of carbon monoxide poisoning-related acute kidney injury according to the Kidney Disease Improving Global Outcomes (KDIGO) criteria. Clin Toxicol. 2018; 56(8):759-64. [DOI:10.1080/155636 50.2018.1424890] [PMID]

[21] Lee FY, Chen WK, Lin CL, Lai CY, Wu YS, Lin IC, et al. Organophosphate poisoning and subsequent acute kidney injury risk: A nationwide population-based cohort study. Medicine (Baltimore). 2015; 94(47):e2107. [DOI:10.1097/ MD.0000000000002107] [PMID] [PMCID]

[22] Aronoff GR. Nonsteroidal anti-inflammatory drug induced renal syndromes. J Ky Med Assoc. 1992; 90(7):336-9. [PMID]

[23] Ejaz P, Bhojani K, Joshi VR. NSAIDs and kidney. J Assoc Phys India. 2004; 52:632-40.

[24] Lim S. Metabolic acidosis. Acta Medica Indonesiana. 2007; 39(3):145-50. http://www.inaactamedica.org/archives/2007/Edisi_3_artcl_10.pdf

[25] Paasma R, Hovda KE, Tikkerberi A, Jacobsen D. Methanol mass poisoning in Estonia: Outbreak in 154 patients. Clin Toxicol. 2007; 45(2):152-7. [DOI:10.1080/15563650600956329] [PMID]

[26] Terada Y, Shinohara S, Matui N, Ida T. Amphetamineindeced myoglobinuric acute renal failure. Japanese J Med. 1988; 27(3):305-8. [DOI:10.1097/00007611-198402000-00035] [PMID]

[27] Adebamiro A, Perazella MA. Recurrent acute kidney injury following bath salts intoxication. Am J Kidney Dis. 2012; 59(2):273-5.[DOI:10.1053/j.ajkd.2011.10.012] [PMID]

[28] Dembek ZF, Smith LA, Rusnak JM. Botulism: cause, effects, diagnosis, clinical and laboratory identification, and treatment modalities. Disaster Med Public Health Prep. 2007; 1(2):122-34. [DOI:10.1097/DMP.0b013e318158c5fd] [PMID]

[29] Loghman-Adham M. Renal effects of environmental and occupational lead exposure. Environ Health Perspect. 1997; 105(9):928-39.[DOI:10.4103/0019-5278.44689] [PMID] [PMCID]
[30] Tian LT, Lei LJ, Chang XL, Jin TY, Zheng G, Guo WJ, et al. Risk assessment of renal dysfunction caused by occupational lead exposure. Zhonghua lao dong wei sheng zhi ye bing za zhi= Zhonghua laodong weisheng zhiyebing zazhi= Chinese Journal of Industrial Hygiene and Occupational Diseases. 2010; 28(3):170

[31] McCrae JC, Morrison EE, MacIntyre IM, Dear JW, Webb DJ. Long-term adverse effects of paracetamol - a review. Br J Clin Pharmacol. 2018; 84(10):2218-30. [DOI:10.1111/ bcp.13656] [PMID] [PMCID]

[32] Mazer M, Perrone J. Acetaminophen-induced nephrotoxicity: Pathophysiology, clinical manifestations, and management. J Med Toxicol. 2008; 4(1):2-6. [DOI:10.1007/BF03160941] [PMID] [PMCID]

[33] Robles-Osorio ML, Sabath-Silva E, Sabath E. Arsenicmediated nephrotoxicity. Renal Failure. 2015; 37(4):542-7. [DOI:10.3109/0886022X.2015.1013419] [PMID] 